\title{
Fluoridbasierter Festelektrolytsensor für die Messung der Schwefelkonzentrationen im Floatglas-Prozess
}

\author{
C. Hecker ${ }^{1}$, E. Krikunova, H.-J. Lang, Y. Joseph \\ Institut für Elektronik- und Sensormaterialien, TU Bergakademie Freiberg, Freiberg, Deutschland \\ ${ }^{1}$ Kontakt: christine.hecker@esm.tu-freiberg.de
}

\begin{abstract}
Zusammenfassung
Festelektrolytsensoren auf Basis von Y-dotiertem $\mathrm{ZrO}_{2}$ haben sich in der Praxis als robuste Sensoren zu Bestimmung von Sauerstoffpartialdrücken bewährt. Ziel dieser Arbeiten ist die Herstellung eines langzeitstabilen und driftfreien Sensors zur kontinuierlichen Messung des Schwefelgehaltes im Zinnbad und Schutzgas für den Floatglas-Prozesses. Hierzu wurden einseitig geschlossene Rohre als Festelektrolytmembranen auf Basis von Y-dotiertem $\mathrm{CaF}_{2}$ sowie La-dotiertem $\mathrm{SrF}_{2}$ entwickelt.
\end{abstract}

Schlagwörter: Schwefelsensor, Gassensor, Festelektrolyt, Fluoridionenleiter, Floatglass

\section{Einleitung}

Flachglas wird heute üblicherweise über den Floatglas-Prozess hergestellt. Dabei läuft das geschmolzene Glas in der Floatbadkammer, die unter Schutzgas (90Vol.- $\% \mathrm{~N}_{2}, 10 \mathrm{Vol} .-\% \mathrm{H}_{2}$ ) betrieben wird, auf ein $\mathrm{Bad}$ aus geschmolzenem Zinn. Dieser Prozess erfolgt bei Temperaturen zwischen $500-1000^{\circ} \mathrm{C}$.

Durch den Stoffaustausch zwischen Glasschmelze und Zinnbad kann sich aus dem im Glas befindlichen Schwefel, welcher während des Läuterns eingetragen wurde, mit dem Zinn verbinden, wobei Zinnsulfid gebildet wird. Verdampftes Zinnsulfid reagiert wiederum unterhalb von $700^{\circ} \mathrm{C}$ mit dem Wasserstoff in der Schutzgasatmosphäre zu

Zinn und Schwefelwasserstoff. Infolge der Reaktionen können Glasfehler durch Zinn- und Zinnsulfidtropfen entstehen.

Eine Kontrolle des Schwefelgehaltes im Zinnbad und Schutzgas könnte daher zu einer Minimierung der Fehler führen. Bis heute gibt es jedoch für derartige Prozessbedingungen noch keinen produktionsreifen, kontinuierlich arbeitenden Schwefelsensor.

Für einen Einsatz unter diesen Bedingungen wurde deshalb in vorangegangenen Arbeiten ein Schwefelsensor auf Basis von Strontiumund Lanthanfluorid mit einer schwefelsensitiven Hilfselektrode entwickelt [1-2]. Der Aufbau dieses ersten Sensormodells, welcher bereits unter industriellen Bedingungen getestet wurde, ist in Abb. 1 dargestellt.

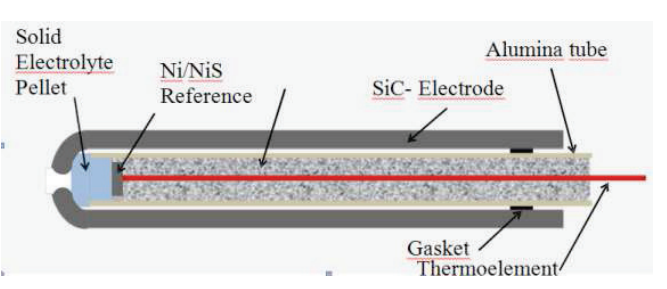

Abb. 1: Schematischer Aufbau des Schwefelsensors

Bei diesem Sensor wurde das Festelektrolytmaterial am Ende eines Korundrohres eingeschmolzen. Hierbei kam es jedoch zu Nebenreaktionen und Ausbildung von Undichtigkeiten, siehe Abb. 2., was sich in einer Zellspannungsdrift während des Einsatzes bemerkbar machte.

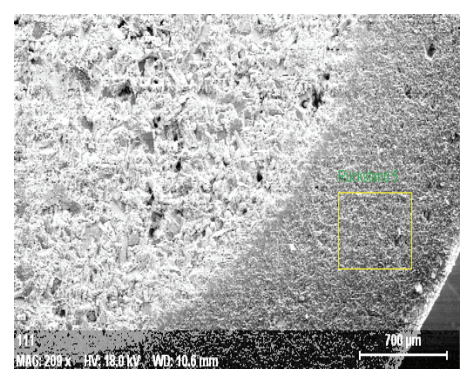

Abb. 2: In Korundrohr eingeschmolzenes, pröses Festelektrolytmaterial

Zur Vermeidung der Reaktionen zwischen Korund und Festelektrolytmaterial soll in einem 
neuen Ansatz die Herstellung des Sensors unter Verwendung einseitig geschlossener

Rohre erfolgen. Hierzu wurden verschiedene Fluoridmaterialien auf ihre Eignung getestet und die Parameter für die Herstellung dicht gesinterter Festelektrolytrohre unter Einsatz der Gießtechnologie ermittelt.

\section{Methoden und Materialien}

Für die Herstellung einseitig geschlossener fluoridischer Festelektrolytrohre wurde die keramische Gießtechnologie unter Verwendung von Gipsformen ausgewählt. Hierzu ist die Herstellung einer wässrigen, stabilen und gießfähigen Suspension mit einem hohem Feststoffgehalt erforderlich. Es wurden Suspensionen aus $\mathrm{CaF}_{2}$ mit bis zu $30 \mathrm{Mol} .-\%$ $\mathrm{YF}_{3}$ sowie $\mathrm{SrF}_{2}$ mit bis zu $30 \mathrm{Mol} . \% \mathrm{LaF}_{3}$ hergestellt, wobei $Y$ und La jeweils als Dotierelemente fungierten.

Die Ausgangssubstanzen und gesinterten Proben wurden mittels röntgenographischer und rasterelektronenmikroskopischer Untersuchungen charakterisiert. Eine Analyse der Partikelgröße der verwendeten Ausgangsmaterialien erfolgte zusätzlich mittels Laserdiffraktometrie und durch Gasadsorptionsmessungen zur Bestimmung der spezifischen Oberfläche (B.E.T). Zur Verbesserung der Gießfähigkeit und Entformbarkeit wurden verschiedene Dispergatoren und Binder getestet und deren Einfluss anhand von Gießversuchen untersucht. Die Suspensionen wurden mittels elektrokinetischer und rheologischer Untersuchungen charakterisiert. Zur Ermittlung eines geeigneten Sinterregimes wurden unterstützend DSC-TG-Analysen durchgeführt. Die Sinterergebnisse wurde mittels Dichtemessungen und anhand von rasterelektronenmikroskopischer

Untersuchungen sowie Bubblepointtests beurteilt.

\section{Ergebnisse}

Durch die Verwendung eines Polyelektrolyten und Binders konnte eine gießähige Suspension hergestellt werden, die es erlaubt, dünnwandige rohrförmige Sensorelemente mit einem Außendurchmesser von $1 \mathrm{~cm}$ herzustellen. Durch den Polyelektrolyten erfolgt eine Ladungsumkehr, siehe Abb. 3, und es entsteht die Ausbildung eines dichtwandigen Körpers, siehe Abb. 4.

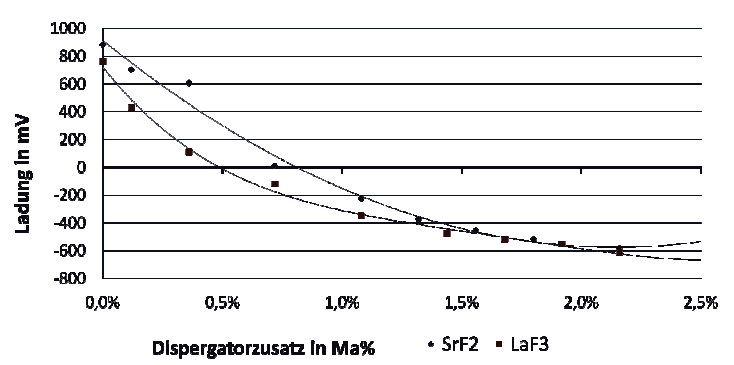

Abb. 3: Einfluss des Polyelektrolyten auf das Strömungspotential als Maß für die Partikelladung

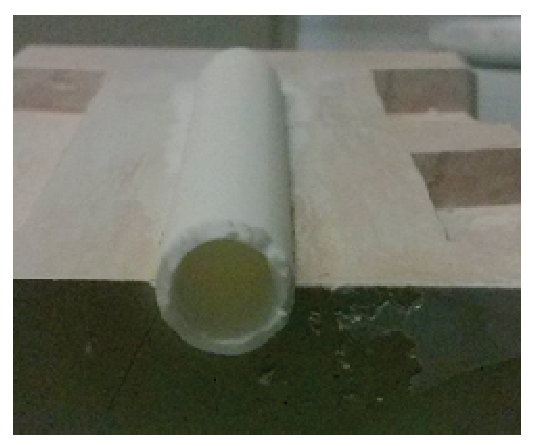

Abb. 4: Gegossenes Sensorelement in der Gipsform vor dem Entformen

Zur Herstellung von dicht gesinterten Rohren wurde ein zweistufiger Sinterprozess erforderlich. In einem ersten Schritt wurden die Rohre unter Luft bei $700^{\circ} \mathrm{C}$ zum Ausbrennen der Organik und zur Verfestigung behandelt.

In Abb. 5 ist die DSC-TG Analyse für eine $0.7 \mathrm{SrF}_{2}-0.3 \mathrm{LaF}_{3}$ Mischung dargestellt. Der Ausbrand der Organik erfolgt danach bis $400^{\circ} \mathrm{C}$.

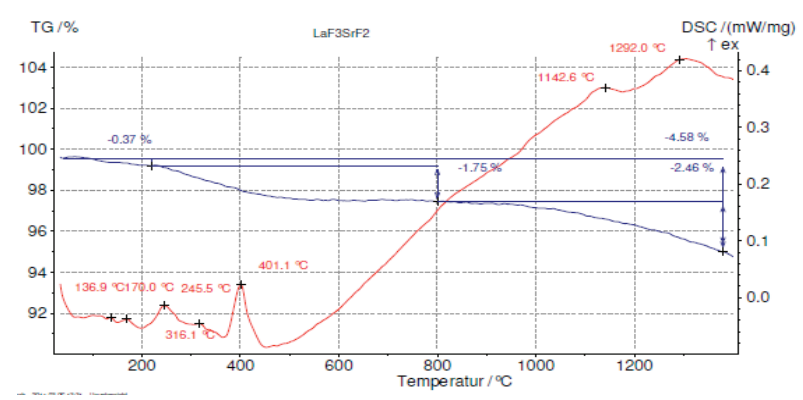

Abb. 5: DSC/TG Analyse für eine Mischung aus $0.7 \mathrm{SrF}_{2}-0.3 \mathrm{LaF}_{3}$ 
Das Dichtsintern der Rohre erfolgte unter Argon bei $1400^{\circ} \mathrm{C}$ mit 2 Stunden Haltezeit für die $0.7 \mathrm{SrF}_{2}-0.3 \mathrm{LaF}_{3}-$ Mischung, siehe Abb. 6.

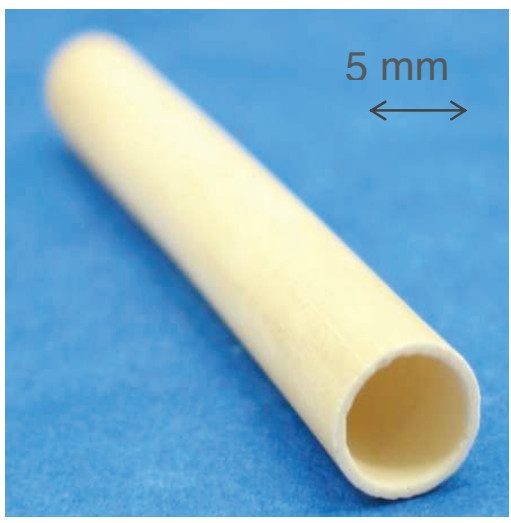

Abb. 6: Gesintertes Rohr aus

$0.7 \mathrm{SrF}_{2}-0.3 \mathrm{LaF}_{3}$

Für die $\mathrm{CaF}_{2}-\mathrm{YF}_{3}$ Rohre ist in $\mathrm{Abb} .7 \mathrm{der}$ Einfluss der Zusammensetzung auf das Sinterergebnis nach einer Sinterung bei $1300^{\circ} \mathrm{C}$ und $2 \mathrm{~h}$ Haltezeit dargestellt. Gemäß Phasendiagramm und DSC-Analyse liegt die Sintertemperatur etwa 10\% unterhalb der erwarteten Schmelztemperatur. Während für die $0.7 \mathrm{CaF}_{2}-0.3 \mathrm{YF}_{3}-$ Mischung die Schwindung bei ca. $13 \%$ lag, betrug diese hingegen für die $0.9 \mathrm{CaF}_{2}-0.1 \mathrm{YF}_{3}-$ Mischung nur ca. 9\%. Einen weiteren Einfluss auf das Sinterergebnis haben die Reinheit und Partikelmorphologie, siehe Probe d) im Vergleich zu b) und c).

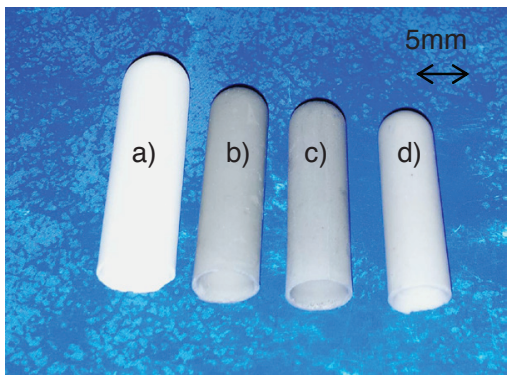

Abb. 7: Rohre aus $\mathrm{CaF}_{2}-\mathrm{YF}_{3}$

a) $0.7 \mathrm{CaF}_{2}$ (99\% Reinheit)- $0.3 \mathrm{YF}_{3}$, vor dem Sintern

b) $0.9 \mathrm{CaF}_{2}$ (99\% Reinheit)- $0.1 \mathrm{YF}_{3}$, nach dem Sintern

c) $0.7 \mathrm{CaF}_{2}$ (99\% Reinheit)- $0.3 \mathrm{YF}_{3}$, nach dem Sintern

c) $0.7 \mathrm{CaF}_{2}\left(99,5 \%\right.$ Reinheit)- $0.3 \mathrm{YF}_{3}$, nach dem Sintern

\section{Diskussion}

Es konnte gezeigt werden, dass über die Gießtechnologie die Herstellung dicht gesinterterte fluoridischer einseitig geschlossener Rohre als Festelektrolytmembran möglich ist. Diese können als Basis für den Bau eines verbesserten Schwefelsensors dienen.

\section{Literatur}

[1] LANG, H.-J.; ELBORG, M.; FANKHÄNL, B. : Development of sulphur sensors for the tin melt and the protective gas. In: The float glass process. Glass Technology - European Journal of Glass Science and Technology Part A 01 52(1), 11-14, 2011

[2] MÜLLER-SIMON, H.; BOEHM, P. : ADAMAIK, M.: Entwicklung eines Sensors für die Schwefelmessung im Schutzgas und der Zinnschmelze einer Floatkammer, DGG Journal 11, Nr. 6, 2012 